\title{
Um novo método para avaliar as inclinações dentárias utilizando a tomografia computadorizada
}

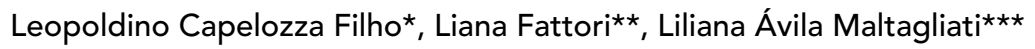

\begin{abstract}
Resumo
O exame de tomografia computadorizada vem sendo amplamente utilizado na Odontologia, principalmente na área de Implantodontia, com o objetivo de mensurar a altura e a espessura ósseas. A possibilidade de realizar cortes tomográficos individualizados nos fez levantar a hipótese de sua aplicabilidade para medir inclinações e angulações dentárias na Ortodontia, com maior precisão e confiabilidade. O objetivo deste trabalho é descrever uma nova metodologia para a mensuração das inclinações e angulações dentárias, utilizando a tomografia computadorizada volumétrica. Para isso, foram realizados cortes tomográficos dos dentes anteriores, de dois indivíduos, com padrões faciais II e III, respectivamente. Após a avaliação, concluímos que a tomografia computadorizada pode ser um meio útil para avaliação de inclinações e angulações dentárias, possibilitando grande contribuição para as pesquisas envolvendo o posicionamento dentário e também para a individualização do tratamento ortodôntico, uma vez que permite a verificação individual do posicionamento dentário.
\end{abstract}

Palavras-chave: Tomografia computadorizada. Inclinações dentárias. Ortodontia.

\section{INTRODUÇÃO}

As inclinações e angulações dentárias são objetos de estudo da Ortodontia desde a época em que Angle, em 1928, sistematizou o tratamento ortodôntico, desenvolvendo o aparelho arco de canto, cujas inclinações e angulações dentárias são modificadas pelos arcos, conforme inseridos nas canaletas dos braquetes ${ }^{8}$.

As inclinações foram estudadas por diferentes autores, que publicaram análises cefalométricas apoiadas em um posicionamento dentário ideal, com o objetivo de propor diferentes condutas ortodônticas (Tweed, Steiner, Ricketts, Interlandi) ${ }^{13}$. As análises cefalométricas também foram empregadas para avaliar alterações decorrentes de diferentes terapias, em estudos comparativos e longitudinais. Andrews ${ }^{2}$, em 1970, inovou a Ortodontia com o desenvolvimento de um estudo realizado em modelos de gesso. Avaliando oclusões ótimas naturais, teve a brilhante idéia de mensurar as inclinações e angulações de todos os dentes superiores e inferiores, trazendo à luz do conhecimento

\footnotetext{
* Professor Doutor da Faculdade de Odontologia da Universidade de São Paulo, USP-Bauru, e membro do setor de Ortodontia do HRAC da Universidade de São Paulo, USP-Bauru. Professor do Programa de Pós-graduação em Ortodontia da Faculdade de Odontologia de Araçatuba - UNESP.

** Mestranda do Programa de Pós-graduação em Odontologia, área de Concentração Ortodontia da Universidade Metodista de São Paulo.

*** Professora Doutora em Ortodontia pela FOB-USP; Professora do Programa de Pós-graduação em Odontologia (Mestrado) - Área de Concentração Ortodontia da UMESP e Coordenadora do curso de Especialização em Ortodontia da UMESP.
} 
o posicionamento dentário individual, em todos os planos do espaço, que culminou na preconização dos braquetes programados, vislumbrando inclusive, individualizações para as diferentes más oclusões dentárias bem como para os casos com e sem extrações. Esta inovação propiciou prever e programar o posicionamento final dos dentes antes mesmo da instalação do aparelho ortodôntico.

Após as prescrições propostas por Andrews ${ }^{3}$, vários autores indicaram alterações e adaptações de algumas inclinações e angulações sem, no entanto, realizarem estudos de mensuração em radiografias ou modelos, mas apenas baseando-se em experiência clínica, comprovada em casos terminados ${ }^{5,15,16}$. A criação dos braquetes individualizados para o tratamento compensatório das más oclusões de Classe I, II e III por Capelozza Filho et al. ${ }^{6}$ teve também a experiência clínica como guia, e esta proposta de individualização tenta, de acordo com os objetivos do tratamento, ajudar a finalização apropriada das angulações e inclinações dentárias destes casos compensatórios.

São escassos os trabalhos, entretanto, que estudam, assim como Andrews fez, as inclinações e angulações dentárias individuais, ou seja, dente a dente, tanto para verificar diferenças entre oclusões normais em diferentes etnias e populações, como para verificar finalização e impressão dos torques e angulações propostos pelas diferentes prescrições.

A metodologia da quase totalidade dos trabalhos se baseia em telerradiografias, em norma lateral e/ou frontal e, em menor quantidade, nas radiografias panorâmicas ${ }^{1,10,18}$. O método radiográfico permite observar o posicionamento dentário manualmente, ou ainda por meio de digitalização das imagens e uso de programas de cefalometria computadorizada. A utilização dos recursos da informática abriu um novo horizonte na Ortodontia, pois além de facilitar o traçado cefalométrico e panorâmico, trouxe a possibilidade de predizer movimentações dentárias, simu- lando o resultado final de um tratamento, inclusive das inclinações e angulações dentárias a serem alcançadas. Seria fantástico se não fossem as limitações impostas pelos métodos bidimensionais, como as radiografias convencionais. Por exemplo, ao utilizarmos telerradiografias laterais, só temos a possibilidade de analisar a inclinação dos incisivos centrais e angulação dos dentes posteriores, ainda com sobreposição de estruturas adjacentes e mesmo de outros dentes. Já na radiografia panorâmica, a distorção nos permite analisar, com certa clareza, apenas as angulações dentárias dos dentes anteriores.

Zanelato $^{19}$ avaliou 60 modelos de gesso de indivíduos brasileiros com oclusão normal natural, com o objetivo de verificar se, na população brasileira, os valores propostos por Andrews seriam corretamente empregados. Utilizou um dispositivo especialmente desenvolvido pelo Departamento de Ortodontia da Universidade Metodista de São Paulo, com o intuito de prover maior confiabilidade às medições das inclinações e angulações dos dentes, já que a tentativa de reproduzir o mesmo dispositivo utilizado por Andrews, trouxe erros de medição de até $5^{\circ} \mathrm{em}$ alguns casos, diferença considerada muito importante do ponto de vista ortodôntico. Bastia ${ }^{4}$ utilizou o mesmo dispositivo para analisar as inclinações e angulações dentárias obtidas com a prescrição MBT, no tratamento ortodôntico sem extrações.

A dificuldade de desenvolver procedimentos metodológicos que permitam a avaliação individual dos dentes é uma das responsáveis pela escassez de trabalhos voltados a descobrir o posicionamento vestíbulo-lingual e mésio-distal dos dentes. A metodologia empregada por Andrews ${ }^{3}$ torna-se complicada e de difícil reprodução, e o dispositivo mencionado e utilizado por Bastia ${ }^{4}$ mesmo sendo um método prático e confiável, é igualmente de difícil reprodução.

A evolução da Imaginologia na Odontologia, no entanto, vem disponibilizando meios diag- 
nósticos precisos, com grande confiabilidade e detalhamento de estruturas em três dimensões. Exemplos disto são os exames tomográficos, cada vez mais utilizados em todas as áreas da Odontologia. A tomografia computadorizada (TC) permite a reconstrução de áreas anatômicas e a visualização em três dimensões, revelando informações sobre tamanho, forma e textura. Tornou-se, assim, um importante instrumento de guia para a Implantodontia, Radiologia e Estomatologia. Na Ortodontia, não é amplamente utilizada, pois traria poucas informações adicionais de diagnóstico, a não ser em casos de suspeita de alguma neoplasia ou impacções dentárias. Porém, com a fabricação do tomógrafo volumétrico e um software específico que permite a realização de mensurações nos cortes tomográficos realizados, surge uma nova possibilidade de metodologia de avaliação das inclinações e angulações, com uma representação fiel das estruturas dentárias. Esta inovação nos motivou a estudar se a TC volumétrica, associada a um software específico, nos permitiria realizar as mensurações de inclinação e angulação dentária, de todos os dentes, de forma confiável, propondo então uma nova metodologia de mensuração, sem limitações, com confiabilidade e rapidez e que nos permitiria avaliar todos os dentes, individualmente, eliminando a complexidade dos estudos em modelos, fazendo crescer o estudo destes posicionamentos dentários tão importantes para o diagnóstico, prognóstico e análise de finalização dos casos tratados ortodonticamente.

\section{Introdução da nova metodologia}

Tomografia Computadorizada (TC)

O último avanço tecnológico em diagnóstico por imagem é a representação digital da anatomia do paciente, exatamente como ela se apresenta. A tomografia computadorizada é o exame de escolha para a análise de componentes ósseos e estruturas dentárias ${ }^{7}$.
As novas gerações de scanners tomográficos computadorizados fornecem uma visão completa das estruturas examinadas, em três dimensões, com alta resolução e baixa radiação aos pacientes.

Recentemente, uma nova geração de tomógrafos computadorizados foi desenvolvida especialmente para a região maxilofacial, o NewTom 9000 (NIM, Verona, Itália), especificamente para a Odontologia, proporcionando imagem volumétrica, como os tomógrafos médicos, porém com uma significante redução da radiação a que o paciente é exposto ${ }^{12,14}$, e com vantagens de uma excelente imagem, de baixo custo e risco, fácil manuseio e, ainda, tempo de exame reduzido ${ }^{11}$.

Além disso, apenas uma sessão para aquisição da imagem tomográfica em volume permite que se façam reconstruções nos mais diversos planos, com o auxílio do software, proporcionando múltiplas imagens, como, por exemplo, o scout lateral e frontal, imagens semelhantes às telerradiografias lateral e frontal; a reconstrução oclusal, panorâmica, transaxial, coronal e ainda as reconstruções em 3D.

A tomografia computadorizada volumétrica fornece detalhada identificação das estruturas anatômicas e inúmeras mensurações em diversos planos, sendo já muito utilizada para diversos fins na Odontologia, como por exemplo, a identificação topográfica de dentes impactados, cistos e processos inflamatórios e tumorais, assim como a investigação das estruturas ósseas da articulação têmporo-mandibular, além de auxiliar o planejamento na Implantologia e o acompanhamento pré e pós-cirúrgico ${ }^{17}$.

$\mathrm{Na}$ Ortodontia, a utilização dos exames tomográficos auxilia no diagnóstico e planejamento de dentes impactados, principalmente caninos; em casos difíceis nos quais a ancoragem deve ser incrementada com elementos adicionais, como os implantes palatinos, ou parafusos bicorticais, avaliando o nível ósseo e checando a direção de inserção; ou ainda para registrar os efeitos no osso alveolar, no pré e pós-tratamento, 
gerados pela movimentação dentária de corpo (translação) $^{9}$.

\section{Descrição da metodologia}

Este trabalho tem a pretensão de contribuir com a Ortodontia, proporcionando uma nova utilidade para a Tomografia Computadorizada Volumétrica, introduzindo uma nova metodologia para a mensuração das inclinações e angulações dentárias. Para exemplificar o método, descreveremos a utilização em dois pacientes selecionados para tratamento na clínica de pós-graduação em Odontologia da Universidade Metodista de São Paulo.

Para a aquisição das imagens tomográficas, foi empregado o Tomógrafo Computadorizado Volumétrico NewTom DVT-9000 (NIM, Verona, Itália) e o software QR DVT-9000, desenvolvido pelo fabricante do tomógrafo, para a reformatação e visualização das imagens.

Em um único scan da região, com um giro de $360^{\circ}$ do tubo de raios $\mathrm{X}$ e seu sensor ao redor da cabeça do paciente, uma imagem em volume da região é adquirida.

A partir do scout lateral, fazemos a reconstrução primária das imagens para obtermos os slices dentro dos parâmetros desejados. Como a inclinação e angulação dentárias são obtidas a partir do plano oclusal, utilizamos este como

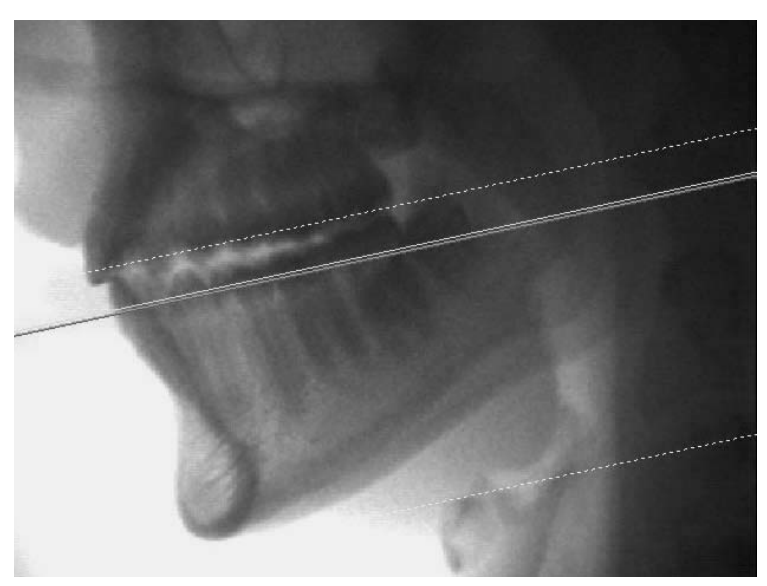

FIGURA 1 - Scout lateral, após reformatação das imagens.

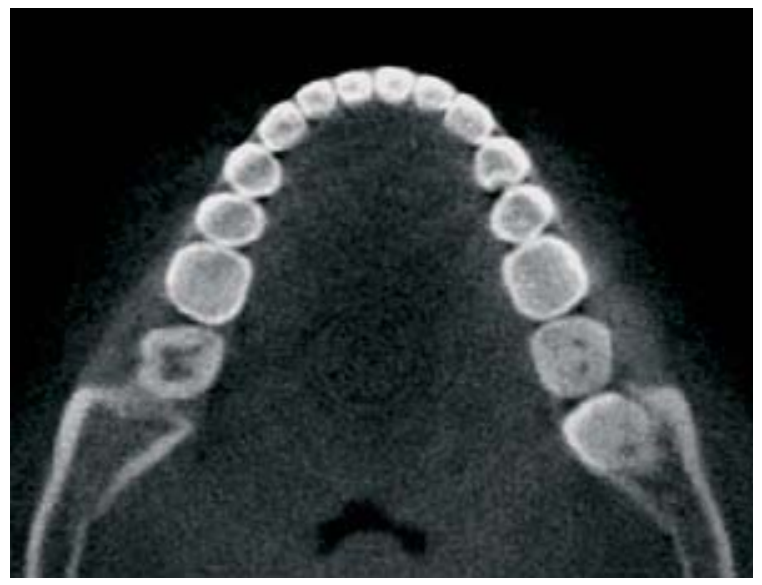

FIGURA 2 - Imagem axial do centro da coroa clínica dos dentes anteriores.

referência para a reconstrução primária da maxila, e depois da mandíbula, de modo que todos os slices tivessem este plano como referência, e fossem paralelos a ele, viabilizando as mensurações (Fig. 1).

Com toda a imagem volumétrica reformatada conforme o plano oclusal, escolhemos os slices próximos ao centro da coroa clínica dos dentes anteriores. Estes slices são representados por linhas coloridas, e possuem $0,3 \mathrm{~mm}$ de espessura. Selecionamos o slice que melhor representa o centro da coroa clínica dentária e a partir dele fazemos a reconstrução secundária da imagem, obtendo uma imagem axial da região (Fig. 2).

$\mathrm{Na}$ imagem axial selecionada, utilizamos ferramentas do software para definirmos a imagem transaxial de cada dente, conforme descrito a seguir.

Com a ferramenta de construção de linha, desenhamos uma reta tangenciando a porção mais vestibular da face vestibular de cada dente anterior a ser estudado, eqüidistante das faces mesial e distal.

Sobre esta linha, com a ferramenta de angulação do software, colocamos o vértice do ângulo sobre o centro da face vestibular e acertamos a angulação em $90^{\circ}$, para obtermos a perpendicular a esta tangente vestibular. Esta reta representa o eixo vestibular da coroa clínica (EVCC). 


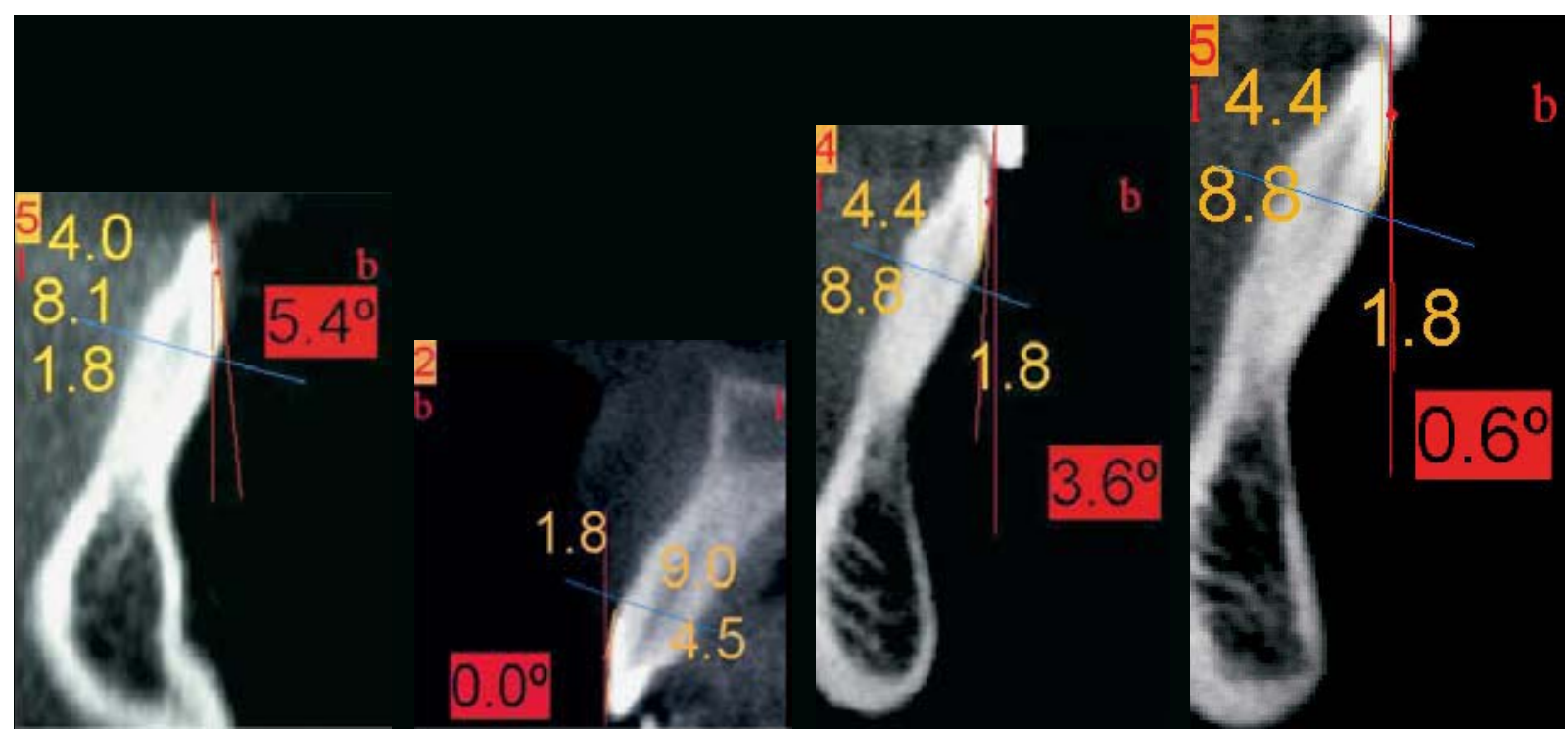

FIGURA 3 - Exemplos de inclinações dentárias.

Sobre esta linha perpendicular, fazemos a reconstrução secundária da imagem, na região exata para a obtenção da imagem transaxial do dente, exatamente no EVCC.

$\mathrm{Na}$ imagem transaxial obtida de cada dente, fazemos os procedimentos, com o auxílio das ferramentas do software para se determinar a inclinação exata de cada dente.

Primeiramente delimitamos a junção amelocementária (JAC), estabelecendo a coroa anatômica do dente. Conforme preconizado por Orban, e citado por Andrews ${ }^{3}$ em sua metodologia, a diferença entre a coroa anatômica e a cora clínica é de 1,8 mm; por isso, subtraímos, a partir da JAC, esta medida da coroa anatômica previamente delimitada, para definirmos a coroa clínica do dente, necessária para a obtenção do ponto do eixo vestibular (ponto EV). Para chegarmos à indicação do ponto $\mathrm{EV}$, localizado no centro da coroa clínica do dente sobre EVCC, utilizamos uma ferramenta que nos dará sua localização exata, a partir da média da distância obtida, entre as extremidades incisal e cervical da coroa clínica. Marcamos o ponto EV, e para avaliarmos a inclinação do dente, utilizamos a ferramenta de angulação do software.

A inclinação exata do dente é dada pelo ângulo formado entre a perpendicular ao plano oclusal, representada pelo limite da imagem, através da reconstrução primária da mesma, e uma tangente e paralela ao ponto EV. Para isso, com o vértice do ângulo sobre o limite da imagem transaxial, ajeitamos um dos seus braços ao ponto EV, e em seguida, ajeitamos o outro, perpendicular ao plano oclusal. Automaticamente, a ferramenta já nos dá a medida obtida. (Fig. 3) Para valores positivos, a extremidade do ângulo, perpendicular ao plano oclusal estará à frente da extremidade passando por EV, e para valores negativos, este braço perpendicular estará atrás da extremidade passando por EV.

$\mathrm{Na}$ tabela 1 temos a exemplificação dos valores de inclinação, obtidos para os dentes anteriores dos dois pacientes examinados.

\section{DISCUSSÃO}

Com os avanços tecnológicos nos exames por imagem, a possibilidade de se ter a melhor visualização da região selecionada, sem distorções, 
Um novo método para avaliar as inclinações dentárias utilizando a tomografia computadorizada

Tabela 1 - Valores de inclinação dos pacientes 1 e 2.

\begin{tabular}{cccc}
\hline \multicolumn{2}{c}{ Paciente 1 } & \multicolumn{2}{c}{ Paciente 2 } \\
\hline Dente & Inclinação & Dente & Inclinação \\
\hline 11 & $9,7^{\circ}$ & 11 & $8,5^{\circ}$ \\
21 & $2,4^{\circ}$ & 21 & $14,6^{\circ}$ \\
12 & $2,5^{\circ}$ & 12 & $11,3^{\circ}$ \\
22 & $5,1^{\circ}$ & 22 & $18,4^{\circ}$ \\
13 & $-4,4^{\circ}$ & 13 & $-5,3^{\circ}$ \\
23 & $-9,6^{\circ}$ & 23 & $-1,4^{\circ}$ \\
31 & $21,8^{\circ}$ & 31 & $-1,2^{\circ}$ \\
41 & $19,2^{\circ}$ & 41 & $-3,6^{\circ}$ \\
32 & $13,3^{\circ}$ & 32 & $-5,4^{\circ}$ \\
42 & $12,2^{\circ}$ & 42 & $-3,3^{\circ}$ \\
33 & $-4,2^{\circ}$ & 33 & $-12,6^{\circ}$ \\
43 & $-7,6^{\circ}$ & 43 & $-7,6^{\circ}$ \\
\hline
\end{tabular}

com baixa dose de radiação ao paciente, sem magnificação, fácil manuseio, e ainda com baixo custo, faz com que cada vez mais profissionais optem pelas tomografias, elevando a qualidade dos exames complementares necessários para um bom diagnóstico ortodôntico.

A substituição dos exames radiográficos convencionais pelos exames tomográficos é uma tendência mundial e que vai fazer cada vez mais parte das rotinas acadêmica e clínica.

Neste trabalho demonstramos a utilização da metodologia para se avaliar as inclinações dentárias por meio de tomografia computadorizada volumétrica. Este estudo das inclinações dentárias abrange muitas áreas da Ortodontia, podendo ser aplicado tanto para a pesquisa científica quanto para estudos clínicos, como por exemplo; no estudo de amostras de pacientes que apresentem oclusão normal natural, e assim, determinar as inclinações médias; bem como no estudo das características de determinadas más oclusões; avaliar a expressão do torque dos braquetes nas diferentes prescrições existentes; investigar o posicionamento inicial e final dos dentes movimen- tados ortodonticamente, e analisar suas posições em relação às respectivas corticais ósseas; comprovação de mecânicas empregadas, como movimentos de corpo ou de pêndulo; verificar a presença ou não de reabsorções radiculares; prever o posicionamento final de alguns dentes quando da utilização de prescrições determinadas; diagnosticar a posição inicial dos dentes dos pacientes que serão submetidos ao tratamento ortodôntico, compensatório ou cirúrgico, e com isso, individualizar a prescrição ideal a ser utilizada, buscando o resultado final ideal.

O exame tomográfico volumétrico é o método de escolha para o estudo das inclinações e angulações dentárias, por ser um método de grande facilidade para a obtenção tanto das imagens quanto das medidas; por apresentar uma confiabilidade superior dos resultados obtidos, em relação aos outros métodos, por não possuir magnificação das imagens adquiridas, e pela sua proporção de 1:1 em relação às estruturas examinadas, não havendo nenhuma distorção, e erros relacionados a isto; e por ser um método bastante versátil, pois, a partir de um exame tomográfico, poderemos obter várias reconstruções, nos mais diversos ângulos e cortes. Além disto, diversos estudos demonstram sua baixa dose de radiação, quando comparada a outros tomógrafos, ou ainda às telerradiografias, tão utilizadas em nosso meio.

Como a utilização da tomografia computadorizada volumétrica para a avaliação das inclinações e angulações dentárias é um exame inovador e superior, quando comparada aos outros métodos existentes, não há dúvidas da sua ampla utilização. 


\title{
A new method to evaluate teeth tipping using computerized tomography
}

\begin{abstract}
Computerized tomography has been widely used in Dentistry, mainly in Implantology, with the purpose of measuring height and thickness of the bone. The possibility of carrying through individual tomographic cuts raised the hypothesis of its applicability on determining dental inclinations and angulations in Orthodontics, with the highest precision and therefore higher trustworthiness. The purpose of this study is to introduce a new method to evaluate teeth tipping, using computerized tomography. For this purpose, tomography cuts of all upper and lower anterior teeth were obtained, of two male patients. The results showed that computerized tomography could be an useful and trustful way to evaluate teeth inclinations and angulations, contributing for the research of orthodontic treatment outcome, since it allows individual analysis of dental position.
\end{abstract}

Key words: Computerized tomography. Teeth tipping. Orthodontics.

\section{REFERÊNCIAS}

1. ALMEIDA, R. R. Estudo ortopantomográfico das inclinações axiais dos dentes anteriores, comparando pacientes tratados ortodonticamente e jovens com oclusão normal. 1999. Dissertação (Mestrado) - Faculdade de Odontologia da USP, Bauru, 1999

2. ANDREWS, L. F. The six keys to normal occlusion. Am J Orthod, St. Louis, v. 62, no. 3, p. 296-309, Sept. 1972.

3. ANDREWS, L. F. Braquetes individuais. Posicionamento do braquete. Posicionamento da canaleta. In:__. Straight wire: o conceito e o aparelho. San Diego: L. A. Wells, 1989. p. 158-170.

4. BASTIA, F. M. M. Estudo das angulações e inclinações dentárias obtidas no tratamento ortodôntico com a utilização da prescrição MBT $^{\mathrm{TM}}$. 2005. 119f. Dissertação (Mestrado em Ortodontia) - Universidade Metodista de São Paulo, São Bernardo do Campo. 2005

5. BENNETT, J. C.; McLAUGHLIN, R. P. As mecânicas de tratamento ortodôntico e o aparelho pré-ajustado. São Paulo: Artes Médicas, 1994.

6. CAPELOZZA FILHO, L. et al. Individualização de braquetes na técnica Straight-wire: revisão de conceitos e sugestão de indicações para uso. R Dental Press Ortodon Ortop Maxilar, Maringá, v. 4, n. 4, p. 87-106, jul./ago. 1999

7. FIROOZNIA, H.; GOLIMBU, C. N.; RAFII, M.; RAUSCHNING, W.; WEINREB, J. C. MRI and CT of the musculoskeletal system. St. Louis: Mosby Year Book, 1992. p. 443-464.

8. GRABER, T. M.; VANARSDALL JUNIOR, R. L. Ortodontia: princípios e técnicas atuais. 2. ed. Rio de Janeiro: Guanabara Koogan, 1996. p. 585-597.

9. GÜNDÜZ, E.; RODRIGUES-TORRES, C.; GAHLEITNER, A.; HEISSENBERGER, G.; BANTLEON, H. Boné regeneration by bodily tooth movement: Dental computed tomography examination of a patient. Am J Orthod Dentofacial Orthop, St. Louis, v. 125, p. 100-106, 2004.

10. HANS, M. G.; KISHIYAMA, C.; PARKER, S. H.; WOLF, G. R.; NOACHTAR, R. Cephalometric evaluation of two treatment strategies for deep overbite correction. Angle Orthod, Appleton, no. 4, p. 255-276, 1994. 1CD-ROM.

11. HATCHER, D. C.; ABOUDARA, C. L. Diagnosis goes digital. Am J Orthod Dentofacial Orthop, St. Louis, v. 125, p. 512-515, 2004.

12. HEILAND, M.; SCHULZE, D.; ROTHER, U.; SCHMELZLE, R. Midfacial imaging using digital volume tomography. Int Cong Ser, Amsterdan, v. 1256, p. 1230-1234, 2003.

13. JACOBSON, A. Radiographic cephalometry: from basic to videoimaging. Illinois: Quintessence, 1995.
14. MAH, J. K.; DANFORTH, R. A.; BUMANN, A.; HATCHER, D. Radiation absorved in maxillofacial imaging with a new dental computed tomography device. Oral Surg Oral Med Oral Pathol Oral Radiol Endod, St. Louis, v. 96, p. 508-513, 2003.

15. McLAUGHLIN, R.; BENNETT, J. C.; TREVISI, H. J. Mecânica sistematizada de tratamento ortodôntico. São Paulo: Artes Médicas, 2002.

16. ROTH, R. H. The Straight-wire appliance 17 years later. J Clin Orthod, Boulder, v. 21, p. 632-642, 1987.

17. SCHULZE, D.; HEILAND, M.; SCHMELZLE, R.; ROTHER, U. J. Diagnostic possibilities of cone-beam computed tomography in the facial skeleton. Int Congr Ser, Amsterdam, v. 1268, p. 1179-1183, 2004.

18. URSI, W. J. S.; ALMEIDA, R. R.; TAVANO, O.; HENRIQUES, J. F. C. Assessment of Mesiodistal axial inclination trough panoramic radiography. J Clin Orthod, Boulder, v. 24, no. 3, p.166-173, 1990.

19. ZANELATO, A. C. T. Estudo das angulações e inclinações dentárias em brasileiros, leucodermas com oclusão normal natural. 2003. Dissertação (Mestrado)-UNIMEP, Piracicaba, 2003. 